\title{
Magnitude-Dependent Transient Increase of Seismogenic Depth
}

\author{
Olaf Zielke ${ }^{* 1}$, Danijel Schorlemmer ${ }^{2}$, Sigurjon Jónsson ${ }^{1}$, and Paul Martin Mai ${ }^{1}$
}

\begin{abstract}
The thickness of the seismogenic zone in the Earth's crust plays an important role in seismotectonics, affecting fault-system architecture and relative fault activity, earthquake size and distribution within a fault system, as well as long-term accumulation of tectonic deformation. Within the last two decades, several studies have revealed that aftershocks of large continental earthquakes may occur below the background depth of the seismogenic zone, that is, below the seismic-aseismic transition zone. This observation may be explained with a strain- and strain-rate-induced shift in rheological behavior that follows large mainshocks, transiently changing the deformation style below the seismogenic zone from incipient ductile to seismically brittle failure. As large earthquakes transiently deepen the seismic-aseismic transition zone, it is plausible to assume that larger mainshocks may cause stronger deepening than smaller mainshocks. Corresponding observations, however, have not yet been reported. Here, we use welllocated seismic catalogs from Alaska, California, Japan, and Turkey to analyze if mainshock size positively correlates with the amount of transient deepening of the seismicaseismic transition zone. We compare the depths of background seismicity with aftershock depths of 16 continental strike-slip earthquakes $(6 \leq M \leq 7.8)$ and find that large mainshocks do cause stronger transient deepening than moderate-size mainshocks. We further suggest that this deepening effect also applies to the mainshocks themselves, with larger mainshock coseismic ruptures being capable of extending deeper into the normally aseismic zone. This understanding may help address fundamental questions of earthquake-source physics such as the assumed scale invariance of earthquake stress drop and whether fault-slip scales with rupture length or rupture width.
\end{abstract}

Cite this article as Zielke, $\mathrm{O}$.,

D. Schorlemmer, S. Jónsson, and P. M. Ma (2020). Magnitude-Dependent Transient Increase of Seismogenic Depth, Seismol. Res. Lett. XX, 1-10, doi: 10.1785/ 0220190392

Supplemental Material

\section{Introduction}

The Earth's crust can be divided into different layers, based on rheological behavior and the potential to generate earthquakes. A simple conceptual model for the upper crust consists of three layers, with a seismogenic layer where earthquakes may initiate that lies in between an upper layer and a lower layer that behave (generally) aseismically (e.g., Scholz, 1988, 2019; Bürgmann and Dresen, 2008). The seismogenic layer is characterized by elastic behavior, for which the tectonic accumulation of strain causes stress buildup that will eventually lead to stress release via seismic brittle failure or frictional instability processes (e.g., earthquakes) once the accumulated stress exceeds the brittle or frictional strength (e.g., Scholz, 2019). The strength of the brittle crust is commonly represented by the Mohr-Coulomb theory and Byerlee's law (Byerlee, 1978; equation 1) in which $\tau_{b}$ is brittle shear stress (i.e., strength), $\mu$ is internal friction coefficient, and $\sigma_{\text {eff }}$ is effective normal stress:

$\tau_{b}=\mu \sigma_{\text {eff }}$
The aseismic regime below the seismogenic layer is characterized by incipient ductile deformation, in which the existing pressure and temperature conditions cause one of the protolith's mineral phases (typically quartz) to deform in a ductile manner (as is exemplified by first appearance of mylonites; e.g., Scholz, 2019). Here, strain accumulation is not associated with a continuous buildup of stress. Instead, stresses remain, once reached, at a certain level that corresponds to the protolith's ductile strength, which, in turn, is controlled by composition, temperature, pressure, and strain rate, and may be represented, for example, as power-law creep (e.g., Turcotte and Schubert, 2002; Ellis and Stöckhert, 2004; equation 2), in which $\tau_{d}$ and $\ddot{\varepsilon}$ are second invariants of deviatoric stress (i.e., representing ductile strength) and strain-rate tensor, respectively, $B$ is a

\footnotetext{
1. Physical Science and Engineering Division, King Abdullah University of Science and Technology (KAUST), Thuwal, Saudi Arabia; 2. Seismic Hazard and Risk Dynamics, Helmholtz-Zentrum Potsdam, Deutsches GeoForschungsZentrum GFZ, Potsdam, Germany

*Corresponding author: olaf.zielke@kaust.edu.sa

(c) Seismological Society of America
} 


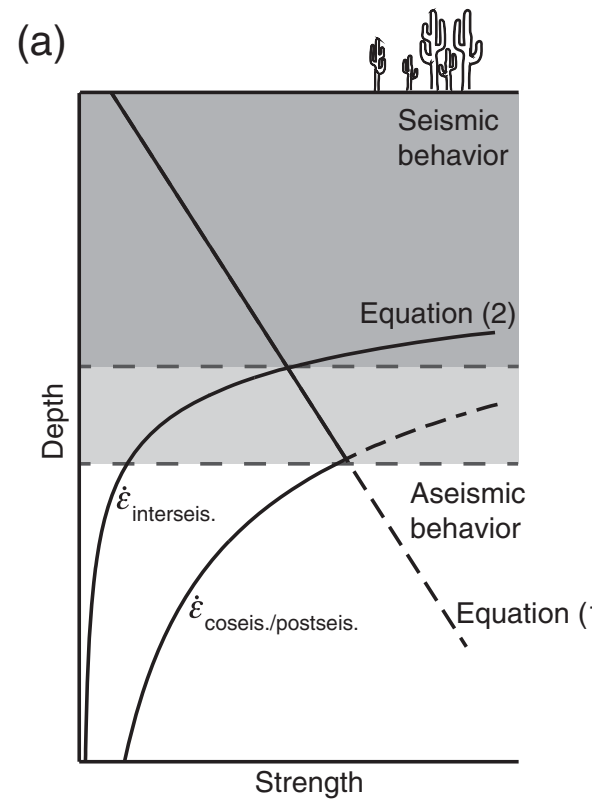

(b)

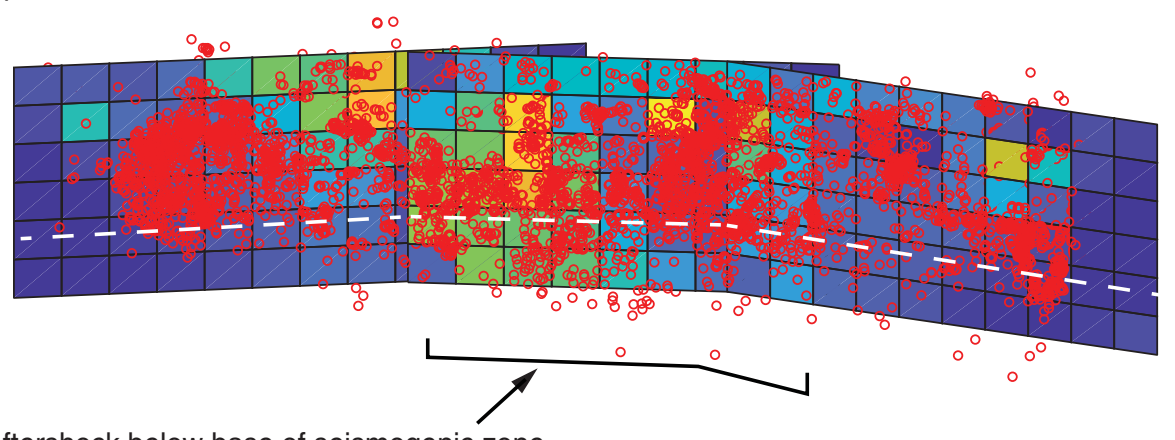

Aftershock below base of seismogenic zone concentrated in area of large coseismic slip
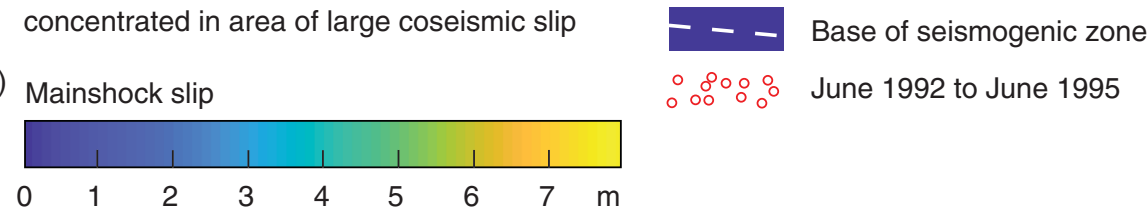

geometrical factor (Ellis and Stöckhert, 2004), $n$ is the power-law exponent, $Q$ is activation energy, $R$ is the universal gas constant, and $T$ is temperature:

$\tau_{d}=B \ddot{\varepsilon}^{1 / n} \exp \left(\frac{Q}{n R T}\right)$.

Although brittle strength increases with depth (due to increasing effective normal stress with depth; equation 1), the ductile strength decreases with depth (primarily due to temperature increase with depth; equation 2). The lower of the two strength values then determines whether crustal behavior is dominantly brittle or ductile (Fig. 1a). The depth range for which this change in rheological behavior occurs (close to the $\sim 300^{\circ} \mathrm{C}$ isotherm where quartz begins to deform plastically), defines the seismic-aseismic transition zone and is also referred to as the brittle-ductile transition zone (BDTZ; e.g., Scholz, 1988; Ellis and Stöckhert, 2004; Rolandone et al., 2004; Cheng and Ben-Zion, 2019). It plays an important role for tectonic and seismic processes and affects numerous characteristics of actively deforming regions, such as fault architecture and relative fault activity, the potential to generate earthquakes, and their maximum size while also influencing the dynamic rupture process and its seismic radiation. It is therefore important for our understanding of earthquake source physics while also providing practical constraints for seismic hazard assessment.

The depth of this transition zone is not constant in space or time but can change rapidly, for example, due to the occurrence of a large earthquake, when coseismic strain rates may become temporarily substantially higher than interseismic rates of, for example, $10^{-12} \mathrm{~s}^{-1}$ (equation 2; e.g., Ellis and
Figure 1. Schematic representation of transient deepening of the seismogenic depth due to occurrence of an increase in strain rate caused by a large earthquake. (a) Strength envelope of upper crust with upper brittle behavior on top (governed by equation 1) and incipient ductile flow below (governed by equation 2). The transition between both rheological regimes is indicated by a dashed line. Increasing the strain rate (e.g., from interseismic to coseismic rates) increases the crust's ductile strength, lowering the corresponding curve. As a result, regions of the crust that deform by ductile flow in interseismic periods may observe brittle failure during coseismic and postseismic times. (b) Oblique view of a slip model for the 1992 Landers earthquake (Wald and Heaton, 1994) and recorded seismicity in the vicinity of the modeled rupture surface between June 1992 (i.e., the occurrence of the Landers earthquake) and June 1995. The dashed white line indicates D95; depth to approximate the background depth of the seismogenic zone. The central part of the rupture surface exhibits the largest amount of (deep) slip and is associated with a concentration of deep (below D95i) aftershocks, suggesting a correlation between slip amount (i.e., earthquake magnitude) and transient deepening of the seismogenic depth.

Stöckhert, 2004). The strain-rate increase causes a transient downward shift of the ductile part of the strength envelope (e.g., Rolandone et al., 2004; Fig. 1a). As a consequence, parts of the lower aseismic layer that would experience incipient ductile deformation during the interseismic phase may become brittle during the coseismic and postseismic phases, if the applied stresses exceed the rock's or fault's brittle or frictional strength so that they may initiate earthquake rupture. This analytical result has been confirmed by field observations, in which microstructural analysis of exhumed fault zones indicate the coexistence and temporal overlap of ductile and brittle deformation features (e.g., Sibson, 1980, 1986; Trepmann and Stöckhert, 2003; Lin et al., 2005; Stewart and Miranda, 
2017). The transient deepening of the BDTZ is also expressed in aftershock depths of large mainshocks. A number of studies have found that these aftershocks may occur distinctly below the pre-earthquake BDTZ depth (e.g., 3-5 km below the background depth of BDTZ) before reverting back to initial depths within a few years after the mainshock (e.g., Schaff et al., 2002; Rolandone et al., 2004; Cheng and Ben-Zion, 2019).

The question that emerges from these findings and that we addressed in this study is whether this transient deepening of the seismic-aseismic transition zone (i.e., the BDTZ), as indicated by aftershock depth distributions, depends on mainshock size. Do aftershocks of large mainshocks occur deeper within the otherwise aseismic crustal regime than those of smaller mainshocks? In other words, do large earthquakes cause a stronger transient deepening of the seismic-aseismic transition zone than smaller earthquakes? This question emerges because it is clear that not only an increase in strain rate but also an increase in absolute strain (and hence stress) is required to cause failure in the transiently brittle domain. Otherwise, mainshocks of any size would be able to cause aftershocks below the seismogenic zone, which has not been observed (e.g., Cheng and Ben-Zion, 2019). Larger earthquakes, in contrast, are associated with larger rupture areas and larger displacements (e.g., Hanks and Bakun, 2008; Thingbaijam et al., 2017) and therefore may induce higher stress over a wider range into the subsurface, including the lower aseismic zone. Hence, it is reasonable to assume that the transient deepening of the seismogenic zone is a function of mainshock magnitude and that it is concentrated in regions where the mainshock experienced high (in a relative sense) fault slip (Fig. 1b).

In this study, we analyze well-located (i.e., relocated) seismic catalogs from Alaska, California, Japan, and Turkey to investigate if this relationship exists. We concentrate our analysis on continental strike-slip faults because their down-dip rupture dimension is less dependent on fault dip (because it is typically subvertical for strike-slip faults) and therefore is more directly linked to the depth of the seismogenic zone. This facilitates a comparison of the transient increase of seismogenic depth with mainshock magnitude between different regions.

\section{Materials and Methods Catalogs}

To conduct this study, we used openly available seismic catalogs from Alaska (National Earthquake Information Center's Comprehensive Catalog, comprising data from the Alaska earthquake center), northern and southern California (Lin et al., 2007; Waldhauser and Schaff, 2008; Waldhauser, 2009; Hauksson et al., 2012), Japan (Japan Meteorological Agency catalog), and Turkey (Bohnhoff et al., 2016). These catalogs were chosen based on availability, resolution, and accuracy (sufficiently low magnitude of completeness $\mathbf{M}_{\mathbf{c}}$ and precise earthquake location), as well as covered time period and spatial extent.
To ensure that our observations for different catalogs are comparable and to avoid potential biases due to differences in catalog resolution, we applied identical requirements on horizontal and vertical location uncertainty (below $\pm 2 \mathrm{~km}$ ) and magnitude of completeness $\left(\mathbf{M}_{\mathrm{c}}=1.4\right)$ to all catalogs. Similar values for location uncertainties and the same method for $\mathbf{M}_{\mathrm{c}}$ estimation (maximum curvature method; Woessner and Wiemer, 2005) have been adopted by previous studies (maximum location uncertainties of \pm 1.5 and $\pm 2.0 \mathrm{~km}$ in Rolandone et al., 2004; Cheng and Ben-Zion, 2019, respectively). That is, earthquake catalogs were reduced to only contain earthquakes with $\mathbf{M} \geq \mathbf{M}_{\mathrm{c}}$ and with location uncertainties below $\pm 2 \mathrm{~km}$. This approach excludes the use of the Alaska catalog, which has a magnitude of completeness of $\mathbf{M}_{c}=1.9$. To also include the Alaska catalog, containing the largest magnitude earthquake in the investigated data set (M 7.8 Denali earthquake), we repeated the analysis as described later using each catalog's own $\mathbf{M}_{\mathrm{c}}$ value (ranging from $0.5<\mathbf{M}_{\mathrm{c}}<1.9$ ) instead of a uniform $\mathbf{M}_{c}$ value.

\section{Mainshock selection}

From each catalog, we first identify strike-slip earthquakes that are sufficiently large to potentially exhibit transient deepening of the seismogenic zone. We chose a corresponding mainshock cutoff magnitude to be $\mathbf{M}=6.0$. This earthquake size was chosen based on scaling relationships that indicate a corresponding minimum source dimension of $\sim 11 \mathrm{~km}$ (e.g., Mai and Beroza, 2000; Hanks and Bakun, 2008; Thingbaijam et al., 2017), which is a first-order minimum bound on seismogenic depth in tectonically active, continental regimes such as the ones that we investigate here. Hence, earthquakes of this size $(\mathbf{M}=6.0)$ or above would have a minimum source dimension that is similar to or larger than the background seismogenic depth and therefore bear the potential to (a) rupture into the aseismic zone, (b) temporarily lower the seismogenic depth, and (c) induce a sufficient stress change at the base of the seismogenic zone to potentially cause aftershocks below the background seismogenic depth. In addition to the size criteria, we require that a sufficiently large number of earthquakes must have occurred within the vicinity of the mainshock ( $N>2000$; counting earthquakes along the mainshock rupture trace above the used $\mathbf{M}_{c}$ value) to provide meaningful measures of the seismogenic depth and its possible transient changes. In effect, the value of $N$ was defined retrospectively, for which we provide additional reasoning in the Determining the Transient Change in Seismogenic Thickness section. Adopting this selection approach, we found 16 mainshocks with enough aftershocks to investigate a possible transient deepening of the seismogenic zone.

\section{Catalog extraction}

For each mainshock, we extracted all earthquakes (both before and after the mainshock) from the catalog that occurred within 


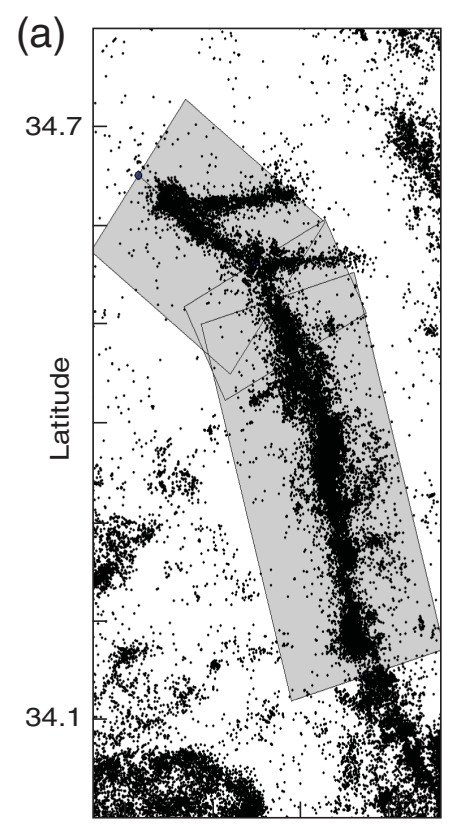

-116.7 Longitude -116.4

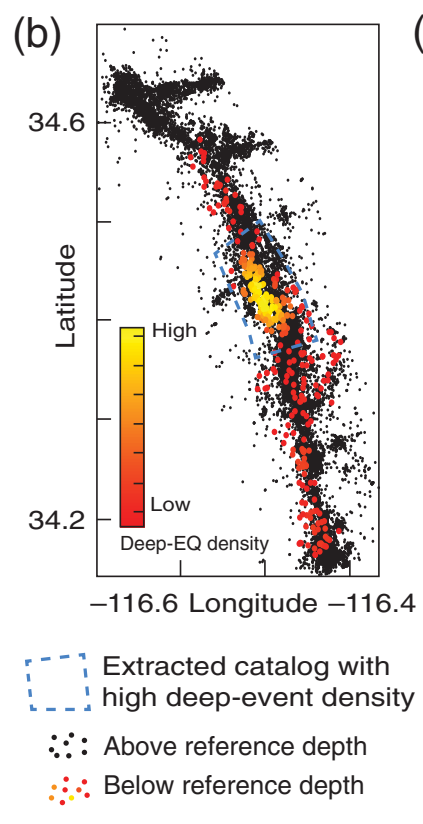

(b)

$\quad$ high deep-event density
$\therefore$ : Above reference depth
$\therefore \therefore$ Below reference depth

hist.

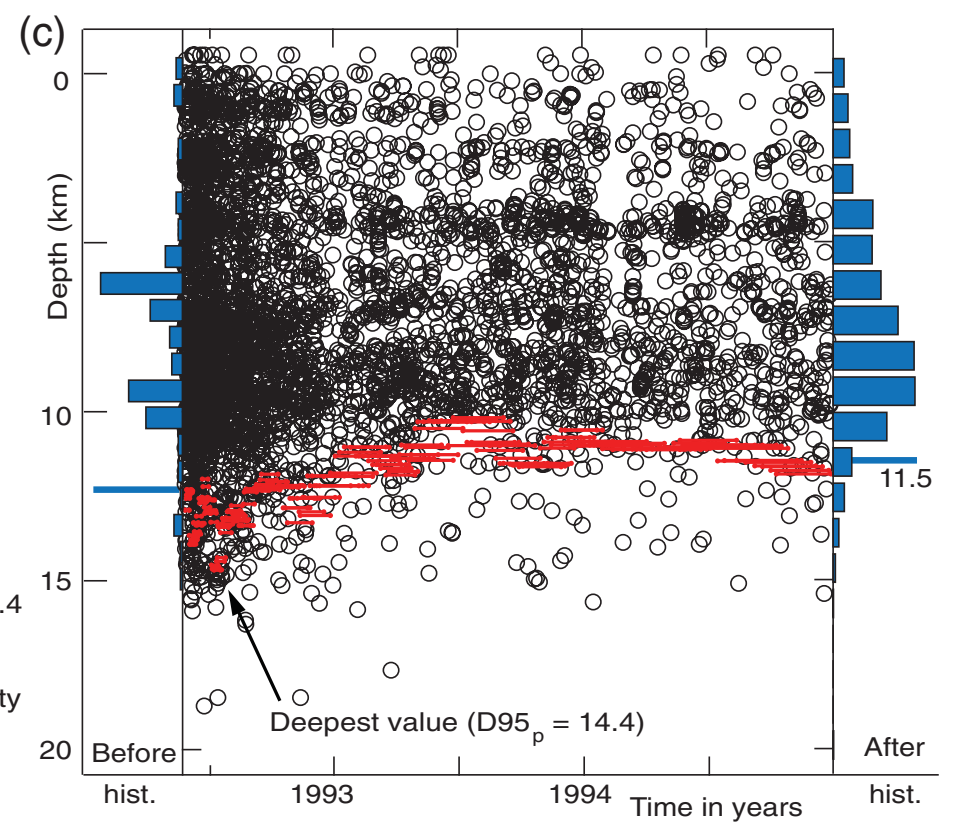

a certain distance (typically $5 \mathrm{~km}$ to either side of the fault, depending on proximity of other faults or earthquake clusters that might interfere with the investigated fault's seismicity) to the fault trace that ruptured during the mainshock. We do this by tracing the fault based on seismicity distribution and source models from kinematic source inversions (e.g., Mai and Thingbaijam, 2014) and extracting the events within a "box" around this fault trace (Fig. 2a). The cropped catalogs may be found in the supplemental material to this article. We estimate the seismogenic depth for this subcatalog following previous studies by computing the D95 depth (depth for which 95\% of earthquakes in the catalog occur above it; Magistrale, 2002; Rolandone et al., 2004; Cheng and Ben-Zion, 2019; Scholz, 2019). This initial estimate of seismogenic depth for the subcatalog is used only to separate earthquakes that occurred above and below this reference depth level (Fig. 2b). Subsequently, we plot all earthquakes from the subcatalog that are equal or deeper than this value with a color-coding that represents the density of deep earthquakes within a $2 \mathrm{~km}$ radius around it (Fig. 2b). In correspondence to the spatial distribution of aftershocks, as well as an earthquake's along-fault slip distribution, this plot (Fig. 2b) exemplifies that the spatial distribution of deep aftershocks is not uniform along the rupture trace but instead may cluster. To identify the maximum postseismic deepening of D95 (as opposed to its average), we (spatially) crop the subcatalog to the along-fault extent for which clusters of deep events have been identified (blue dashed box, Fig. 2b). The actual analysis on transient deepening of the seismogenic zone is then performed on this cropped catalog that contains all earthquakes within the cropping polygon, regardless of their depth or time relative to the mainshock occurrence.
Figure 2. Data selection and analysis steps for the example of the 1992 Landers earthquake. (a) A subcatalog, centered on the mainshock rupture trace is extracted using overlapping boxes of about $10 \mathrm{~km}$ width. (b) We compute the D95i depth for this subcatalog and color-code the density of deep events (those occurring below $\mathrm{D} 95_{\mathrm{i}}$ ) within a $2 \mathrm{~km}$ radius for each deep event. This step allows identifying areas where deep events are clustered. A polygon is drawn around sections where these clusters occur to extract the corresponding events from the catalog. (c) The extracted catalog is split into three time windows: a first time window containing all earthquakes that occurred before the mainshock, a second time window containing the mainshock and all earthquakes occurring during the following half year, and a third time window containing all earthquakes occurring after the second time window. Histograms of depth distribution for the "before" and "after" regions are plotted, and the corresponding D95; value is determined (blue line). The histograms highlight the temporal differences in catalog density and the need to combine "before" and "after" time windows for D95; determination. For the time window with mainshock $+0.5 \mathrm{yr}$, we compute D95 $p$ values for bins of 200 events, using an overlap of $90 \%$ (red lines). We identify the deepest D95 value as well as the consistent depth for $\mathrm{D} 95_{i}$ to determine the transient change of the seismogenic depth. EQ, earthquake.

\section{Determining the transient change in seismogenic thickness}

From the cropped earthquake catalog, we take all earthquakes that occurred before the mainshock and those that occurred at least $0.5 \mathrm{yr}$ after the mainshock to determine the depth of the "interseismic" seismogenic zone, D95 $5_{\mathrm{i}}$, of the cropped region, providing a local measure on background seismogenic depth. The events within this $0.5 \mathrm{yr}$ time window are used to compute the postseismic depth of the seismogenic zone $\mathrm{D} 95_{\mathrm{p}}$. We have 
(a) M 6.7 Noto earthquake

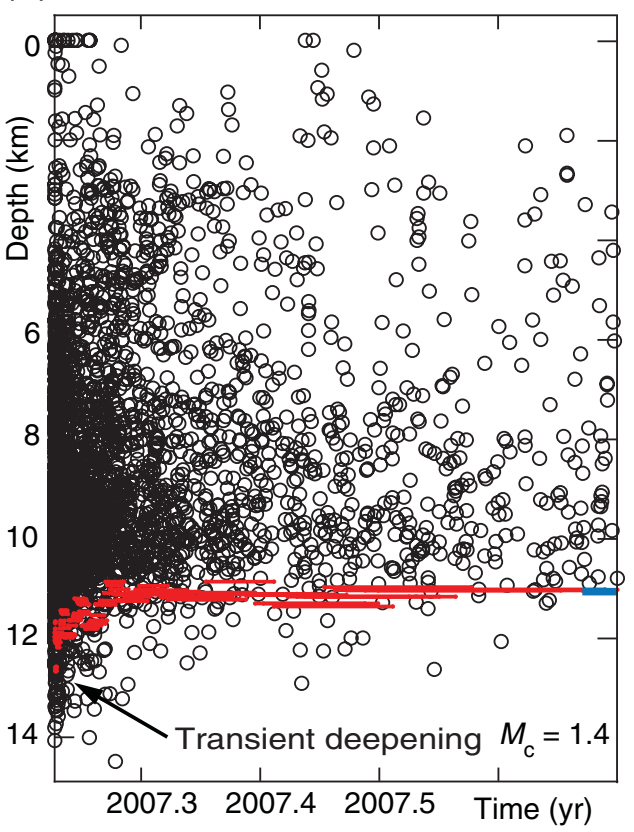

(b) M 7.1 Hector Mine earthquake

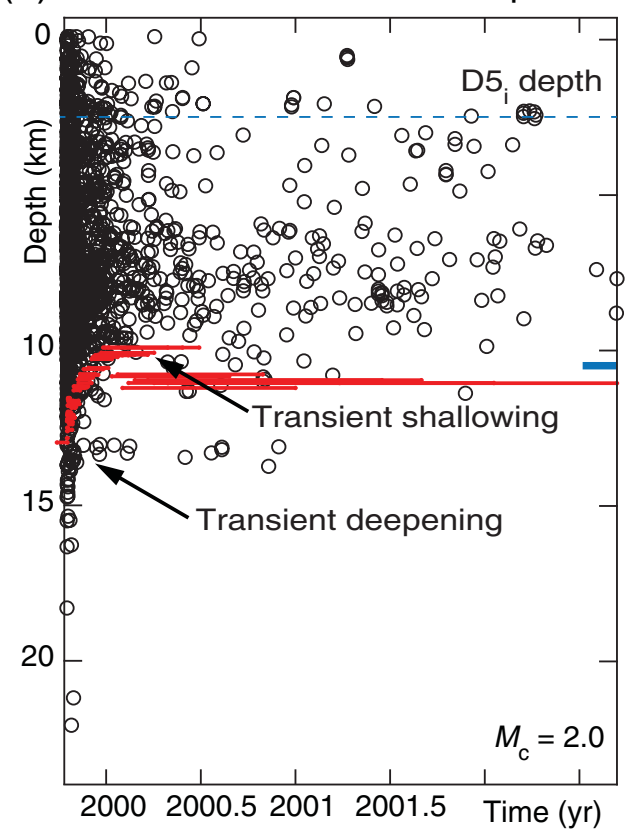

applicable. Two exemplary earthquakes (Fig. 3) appear to

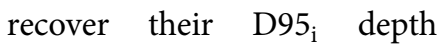
within the first year after the mainshock, substantially faster than the 3 yr time interval. We therefore note that using the 0.5 yr time window is (a) necessary to have a sufficient number of earthquakes in the interseismic and postseismic time windows, (b) permissible, given the observed variations in $\mathrm{D} 5_{\mathrm{i}}$ recovery time, and (c) providing a lower bound on transient deepening. For the extracted $0.5 \mathrm{yr}$ time window (mainshock occurrence time plus $0.5 \mathrm{yr}$ ), we take bins of 200 events with a shift of 20 events to determine the postseismic change in seismogenic depth by computing the corresponding D95 value for each bin. The temporal extent of these bins therefore varies with time, depending on earthquake density through time (Fig. 3). We also performed our analysis using bins of 100 and 400 events with a shift of 10 and 40 events, respectively, to test

chosen the $0.5 \mathrm{yr}$ time window because we are primarily concerned with the maximum D95 $\mathrm{p}$ depth increase, which has been observed to follow more or less directly the mainshock occurrence (e.g., Schaff et al., 2002; Rolandone et al., 2004; Cheng and Ben-Zion, 2019; Figs. $2 c$ and 3). Thus, the maximum transient deepening is contained within this $0.5 \mathrm{yr}$ time window. Further, using this relatively short time window was necessary to ensure that a sufficiently large number of earthquakes lies not only within but also outside of this window, which is necessary for a reliable estimation of $\mathrm{D} 95_{\mathrm{p}}$ as well as $\mathrm{D} 95_{\mathrm{i}}$. That said, independent observations, for example, for the 1992 Landers earthquake have shown that the transient deepening may last for $\sim 3 \mathrm{yr}$ (e.g., Rolandone et al., 2004). It is therefore possible that our D95 $5_{i}$ estimate contains some transient deepening signal if the $0.5 \mathrm{yr}$ time window is indeed substantially shorter than the corresponding $\mathrm{D} 95_{\mathrm{i}}$ recovery period. In that case, our $\mathrm{D} 95_{\mathrm{i}}$ estimate would be biased to be too deep, which means that the transient deepening that we report is a lower bound on the actual transient deepening that occurred. That said, we also want to point out that the previously reported $\sim 3 \mathrm{yr}$ recovery time may not be universally for any dependency on bin size (see Fig. S1). To ensure a sufficient number of events in the interseismic and postseismic time windows (i.e., to fill the largest used bin size), we found that respective catalogs needed to contain a combined amount of $N>2000$ events. The binning approach itself is taken to avoid potential sampling bias due to the larger number of events following directly the time of the mainshock (e.g., Rolandone et al., 2004). The difference between the respective values represents the magnitude of the transient change in seismogenic depth, $\Delta \mathrm{D} 95=\mathrm{D} 95_{\mathrm{p}}-\mathrm{D} 95_{\mathrm{i}}$.

\section{Results \\ Single event, looking at typical shape of time versus depth (shallow vs. deep effect)}

Before discussing the main part of the results, we like to draw attention to common features observed in the transient deepening of the seismogenic zone (Fig. 3). In most cases, the transient deepening directly follows the mainshock occurrence, and soon after that seismicity resumes to its background depth, as is indicated by D95 (Fig. 3a). This is, however, not always the case. After the initial deepening of $\mathrm{D} 95_{\mathrm{p}}$ due to mainshock 
occurrence, we find that $\mathrm{D} 95_{\mathrm{p}}$ may not directly recover to D95 $5_{\mathrm{i}}$, but instead exhibits some form of "overshooting," for which the seismogenic width transiently becomes shallower than $\mathrm{D} 5_{\mathrm{i}}$ before reverting back to the background $\mathrm{D} 95_{\mathrm{i}}$ depth (Fig. 3b). This behavior was observed for three mainshocks. Although the initial transient deepening is expected for sufficiently large mainshocks, as discussed earlier, the transient shallowing requires a different explanation, if it is not simply a result of chance. Therefore, to hypothesize on potential causes for this observation, we offer two possible explanations.

1. First, it is plausible to assume that the coseismically induced changes in frictional instability or rheological behavior affect not only the lower aseismic zone (e.g., Scholz, 2019) but also the upper aseismic zone, albeit in a different manner and via different underlying physical processes. As a consequence, additionally to the deepening of the base of the seismogenic depth due to mainshock occurrence, we may observe a shallowing of the top of the seismogenic depth (Fig. 3b). The latter finds its expression as surface rupture of the mainshock and in higher seismicity rates at shallow depths, transiently pulling the respective boundary (represented here by the 5 th percentile of earthquake depth, $\mathrm{D} 5_{\mathrm{i}}$ ) closer toward the surface. Given the distinctly different conditions at the top and base of the seismogenic zone and the certainly different physical processes that cause these transient signals, we speculate that the rate by which the respective transient signal decays also differs. Hence, the observed transient shallowing of the seismogenic zone that follows the initial transient deepening (Fig. 3b) could be an effect of different rates by which the respective transient signals decay (for which the deep transient signal decays faster than its shallow counterpart, pulling D95 temporarily toward shallower depth levels).

2. Another potential explanation is the occurrence of shallow earthquake clusters that follow the mainshock, pulling D95 temporarily toward shallower depth levels. The sensitivity to such clusters would be particularly high when the aftershock catalog is sparse, for example, due to a relatively high $\mathbf{M}_{\mathrm{c}}$ value.

\section{Transient change of the seismogenic depth}

Following the procedure outlined in the Materials and Methods section, we identified 16 continental strike-slip earthquakes within the analyzed catalogs. These earthquakes range from the smallest one with M 6.0 (2004 Parkfield) to the largest with M 7.8 (2002 Denali). Table 1 presents our estimates of $\mathrm{D} 95_{\mathrm{i}}, \mathrm{D} 95_{\mathrm{p}}, \Delta \mathrm{D} 95_{\text {as }}$ well as the corresponding $\mathbf{M}_{\mathrm{c}}$ value, earthquake number used in the analysis, and mean earthquake slip along the investigated fault section(s). With the exception of the M 6.7 Tottori and possibly the M 6.0 Parkfield earthquakes (both exhibiting $\Delta \mathrm{D} 95$ values close to zero), we find that $\Delta \mathrm{D} 95$ is positive, meaning that the seismic-aseismic transition zone did, in fact, increase in depth during the postseismic phase before reverting back to the $\mathrm{D} 95_{\mathrm{i}}$ depth. A graphical representation of this data set indicates a scattered yet clear positive and approximately linear relationship between earthquake magnitude and transient depth increase of the seismogenic zone (Fig. 4a,b and Fig. S1). Figure 4c and $4 d$ shows $\Delta \mathrm{D} 95$ as a function of mean slip (mean slip along the fault section for which transient deepening was observed) as derived from available kinematic source models (SRCMOD database; Mai and Thingbaijam, 2014) or earthquake scaling relationships (for two earthquakes without published kinematic source model; e.g., Wells and Coppersmith, 1994). Again, we observe a clear, yet somewhat scattered, and approximately linear relationship between both parameters. It reveals that transient deepening is larger in places where more coseismic slip occurred during the mainshock. It also helps explain why, for example, the transient deepening of the M 7.8 Denali earthquake is similar to that of the M 7.3 Landers earthquake: the average slip amounts in the investigated regions (with 2.5 and $2.7 \mathrm{~m}$, respectively) are likewise similar (Table 1 ).

The relatively large amount of scatter in Figure 4 and Figure $\mathrm{S} 1$ is not surprising, given the simplicity of our conceptual model (Fig. 1a) and the variety of regions and data sets that we compare (with different geologic properties and seismotectonic histories). In light of the potentially distinct differences between these regions, we find it intriguing that the assumed relationship between earthquake magnitude (i.e., mean slip) and transient deepening of the seismogenic zone does present itself as clearly as it does.

To further test the sensitivity of our findings with respect to $\mathbf{M}_{\mathfrak{c}}$, we provide $\Delta$ D95 estimates using a uniform $\mathbf{M}_{\mathrm{c}}$ value for all catalogs $\left(\mathbf{M}_{c}=1.4\right)$ as well as $\Delta \mathrm{D} 95$ estimates using catalog-dependent $\mathbf{M}_{\mathrm{c}}$ values (Table 1, values in parentheses). Although discrepancies between both measurements can be observed, we find that the positive correlation between mainshock magnitude and $\Delta \mathrm{D} 95$ is clearly recognized in both and well described by a linear relationship. We further tested the sensitivity of our results with respect to bin size. Figure S1 provides graphical representations for 100- and 400-event bin sizes. The positive, linear relationship between magnitude and $\Delta \mathrm{D} 95$ is also for these bin sizes well established and clearly recognizable. We therefore firmly observe that the amount of transient deepening that follows an earthquake mainshock is proportional to the mainshock's magnitude.

In addition, we performed linear regression (using robust bisquare regression) on our observations (Fig. 4 and Fig. S1). Table 2 summarizes the corresponding parameters. Two aspects are noteworthy. First, as an expression of the observed distinct data scatter, we find that the $R^{2}$ values are overall low. It is important to note that low $R^{2}$ values do 
TABLE 1

Summary of the Results of This Study, Providing Earthquake Name and Year of Occurrence, Catalog Used to Analyze It, Mainshock Moment Magnitude M, and Average Slip along Fault Sections That Exhibited Clusters of Events below D95;

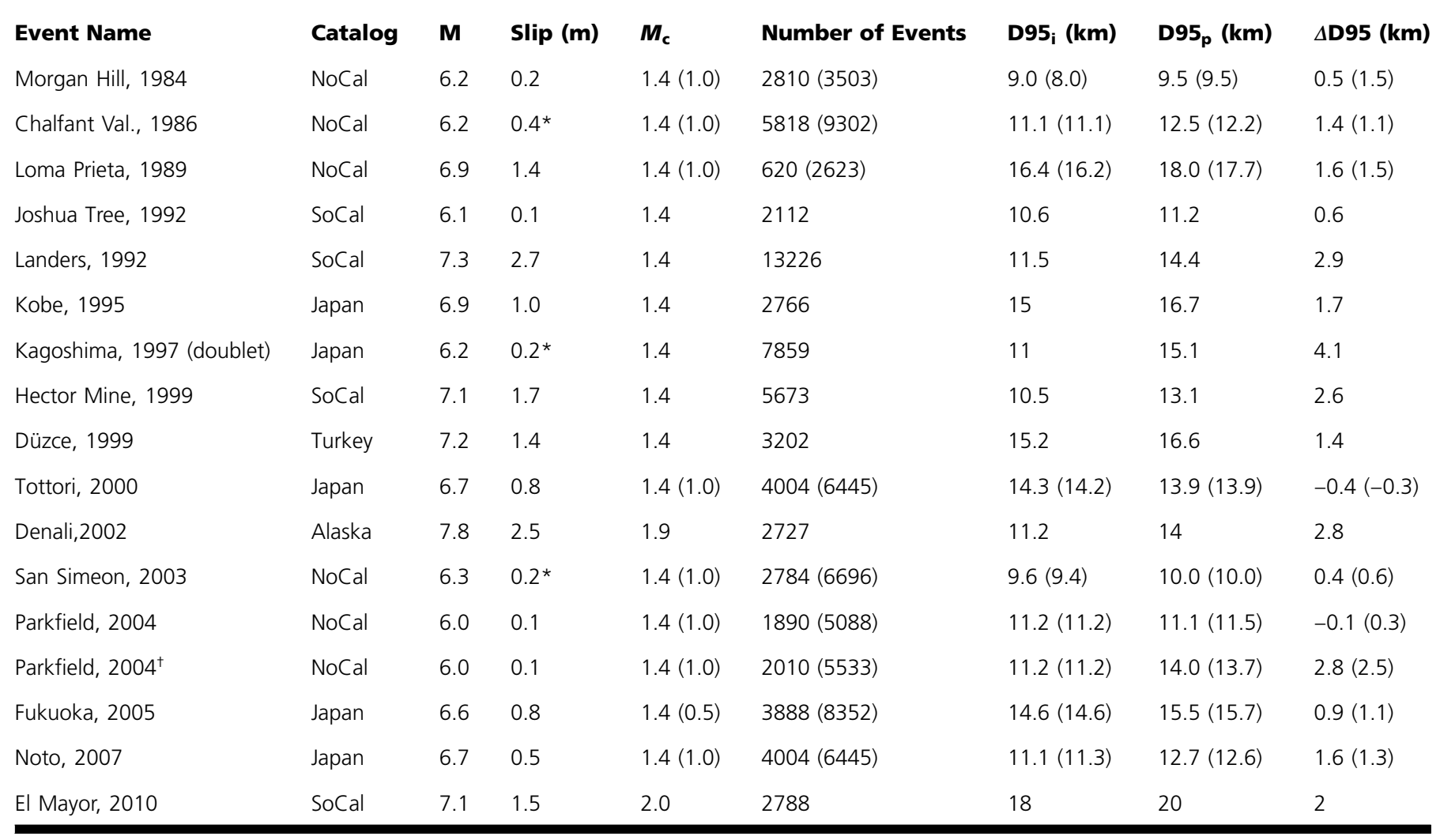

Average slip was computed by averaging results from kinematic source models, accessed via the SRCMOD database (Mai and Thingbaijam, 2014). We also listed magnitude of

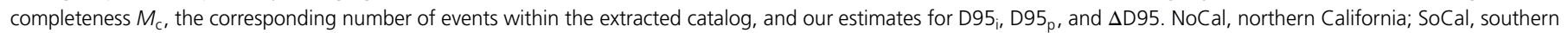
California.

*In these three cases, no source models are publically available, and we used scaling relations from Wells and Coppersmith (1994) to provide a corresponding estimate. tThis measurement includes a deep earthquake cluster that followed shortly after the 2004 Parkfield earthquake.

not imply that these regressions are statistically insignificant, but rather that data are highly scattered (presumably due to differences in geologic properties and tectonic histories of the investigated faults and other epistemic uncertainties). Second, regression values for 100- and 200-event bins exhibit similar slope and intercept values, implying that these regressions recover the underlying physical process equally well. However, slope and intercept are distinctly different for the 400 -event bin realization (Table 2), presumably because these bins are too large (with respect to $\mathbf{M}_{\mathrm{c}}$ and consequently the amount of events in the catalog), therefore masking the transient deepening effect by incorporating too many events that do not exhibit this behavior. The variations in regression results reveal the sensitivity of the analysis to event bin size and indirectly also to $\mathbf{M}_{\mathbf{c}}$. Therefore, they should not be taken literally, but rather be viewed as evidence of an approximately linear relationship between magnitude and transient deepening of the seismogenic zone, regardless of adopted bin size. That is, qualitatively, the regressions all show the same behavior. In summary, transient deepening of the seismogenic zone is positively correlated with mainshock magnitude, that is, mean slip in the region where transient deepening was observed.

\section{Discussion}

We investigate whether mainshock magnitude affects the amount of transient deepening of the seismic-aseismic transition zone. Analyzing the aftershocks of 16 continental strikeslip earthquakes, we identify a clear positive relationship between mainshock magnitude (i.e., mean slip) and the amount of the transient deepening of the seismogenic zone. Large mainshocks tend to generate deeper aftershocks than small mainshocks, that is, large mainshocks lower the seismogenic zone transiently more than smaller mainshocks. While an intuitive result, the presented study is the first to provide observational data to support it.

We hypothesize that not only the aftershocks rupture below the interseismic seismogenic depth, but also the mainshock itself (if sufficiently large) may have done so as well (e.g., King and Wesnousky, 2007). This conjecture-considering 

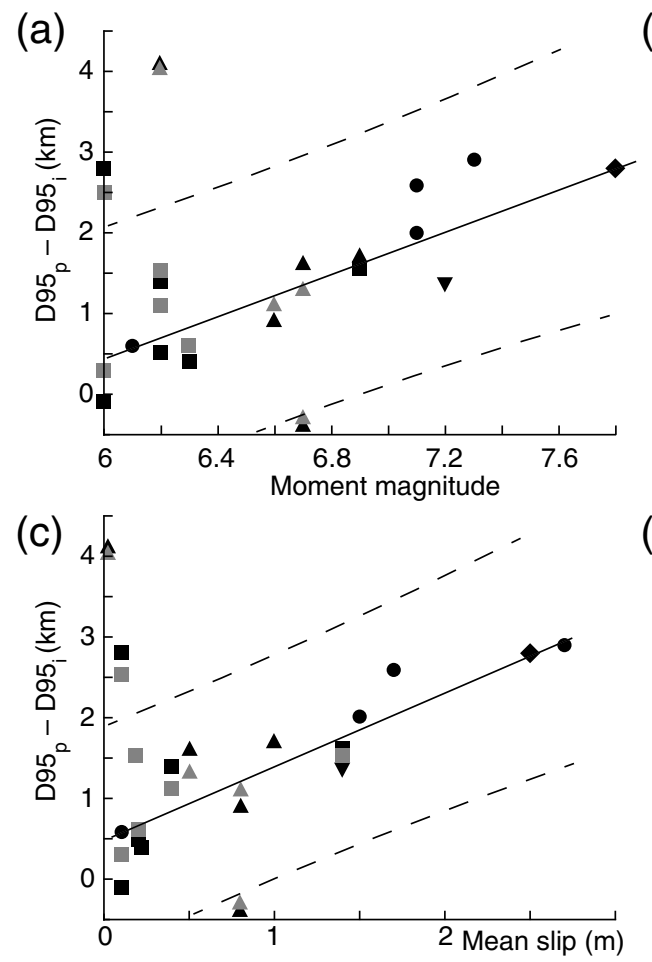

(b)

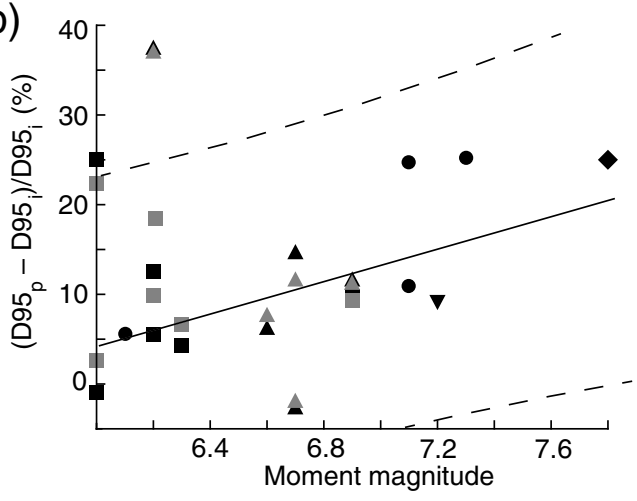

(d)

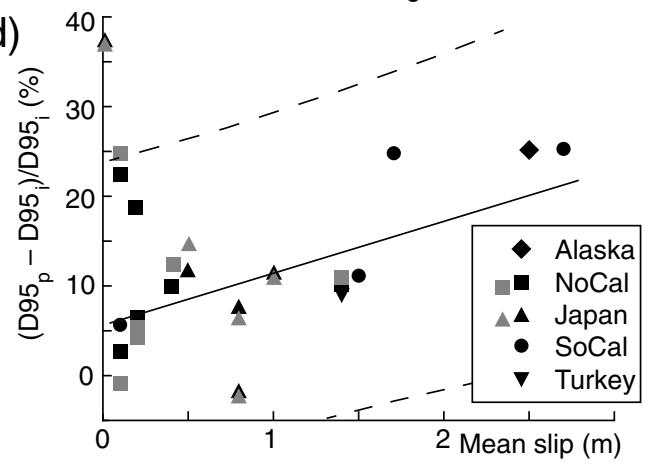

Figure 4. Relationship between mainshock size and increase in seismogenic depth, as indicated by change from $D 95_{i}$ to $D 95_{p}$. All data points from Table 1 are presented and symbols indicate the corresponding catalog while the shading indicates the used $\mathbf{M}_{c}$ value (gray for $\mathbf{M}_{c}=1.4$; black for catalog-dependent $\mathbf{M}_{\mathrm{c}}$ value). (a) Relationship between magnitude and absolute change of seismogenic depth. (b) Relationship between magnitude and relative change of seismogenic depth. (c) Relationship between mean slip and absolute change in seismogenic depth.

(d) Relationship between mean slip and relative change in seismogenic depth. The presented observations indicate a clear positive correlation between mainshock magnitude (i.e., fault slip) and the transient change in seismogenic depth that follows the mainshock. We also plot regression lines (including 95\% confidence intervals), corresponding parameters are listed in Table 2. NoCal, Northern California; SoCal, Southern California.

also that earthquakes may rupture below the interseismic seismogenic zone by an amount that depends on the size of these earthquakesmay shed light on outstanding questions related to earthquake stress drop, the apparent breakdown of self-similarity (whether small and large earthquakes exhibit the same stress drop and source physics), and the discussions how rupture dimension scales with fault slip (e.g., Scholz, 1982, 1994; Romanowicz, 1992; Mai and Beroza, 2000; King and Wesnousky, 2007; Hanks and Bakun, 2008; Allmann and Shearer, 2009; Shaw, 2009; Thingbaijam et al., 2017). For the latter, two end-member models have been formulated in the past to estimate how earthquake slip changes as a function of rupture dimension, namely the $W$ and $L$ models (which stand for scaling dominated by fault width $W$ or fault length $L$, respectively; e.g., Scholz, 1982; Romanowicz, 1992). Although the former assumes that slip saturates

TABLE 2

Parameters for Linear Regressions in Figure 4 and Figure S1

\section{Figure Name}

Figure $4 a$

Figure $4 b$

Figure $4 c$

Figure $4 d$

Figure S1a

Figure S1b

Figure S1C

Figure S1d

\section{Plotted Parameter}

$\mathbf{M}$ vs. $\Delta \mathrm{D} 95,200-$ bin

M vs. $\Delta \mathrm{D} 95 \%, 200$-bin

Mean slip vs. $\Delta \mathrm{D} 95,200$-bin

Mean slip vs. $\Delta$ D95\%, 200-bin

M vs. $\Delta \mathrm{D} 95,100-$ bin

M vs. $\Delta \mathrm{D} 95,400$-bin

M vs. $\Delta \mathrm{D} 95 \%, 100$-bin

M vs. $\Delta \mathrm{D} 95 \%, 400-$ bin

\section{Regression Equation, $f(x)=a x+b$}

$1.3 x-7.4$

$8.9 x-49.2$

$0.9 x+0.5$

$5.8 x+5.7$

$1.6 x-9.0$

$0.5 x-2.8$

$9.3 x-47.6$

$3.5 x-16.1$

\section{Parameter Range}

$R^{2}$

a: $0.6,1.9 ; b:-11.7,-3.1$

0.54

a: $1.5,16.4 ; b:-98.4,0.0$

0.25

a: $0.5,1.3 ; b: 0.1,0.9$

0.65

a: $1.1,10.5 ; b: 0.7,10.7$

0.28

a: $0.9,2.3 ; b:-13.8,-4.2$

0.70

a: $-0.25,1.22 ; b:-7.2,2$.

0.33

a: $1.0,17.6 ; b:-102,2.6$

0.55

a: $-3.2,10.2 ; b:-60.4,28.3$

Regression coefficients (slope and intercept) for 100- and 200-event bins (Fig. 4a,b and Fig. S1a,c) are within the same range while coefficients for the 400-event bin regression are distinctly different. As discussed in the Transient change of the seismogenic depth section, this variation in regression parameters reflects the sensitivity to the number of events combined during binning to determine $\Delta \mathrm{D} 95$, for which larger bins tend to average out the otherwise observed transient deepening. The $R^{2}$ value indicates that observations are scattered. 


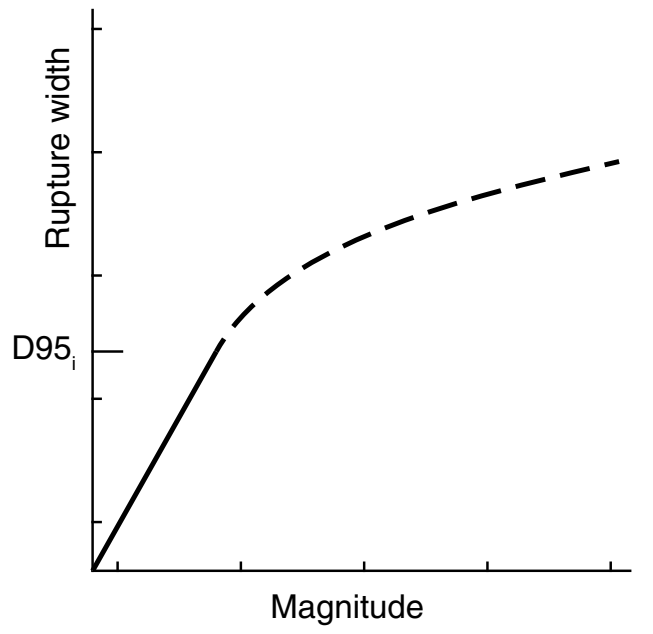

Figure 5. Schematic representation of the suggested changing growth rate of earthquake rupture width as function of earthquake magnitude. Once the rupture width $W$ exceeds the depth of the seismogenic zone (D95i), it becomes increasingly difficult (requiring additional strain) to further increase $W$. As a result, for a given magnitude, the rupture area is smaller with respect to the slip along that area once $W$ has distinctly surpassed $D 95_{i}$.

once an earthquake's smaller rupture dimension equals the fault's width (i.e., the thickness of the seismogenic layer), the latter assumes that slip will continue to grow as a function of fault length. Whether the $L$ or the $W$ model provides the more accurate representation of the underlying physics is still an open debate. Observations have shown that slip does increase even after $W$ is fully ruptured (hence disagreeing with the $W$ model) and by that raised questions about differences in source physics and associated stress drop among large earthquakes (as implied by the $L$ model). At the same time, slip exhibits a distinctly lower growth rate once an earthquake's rupture length exceeds a certain threshold value (Romanowicz, 1992; Scholz, 1994). The observations presented here may indicate a pathway to resolve this apparent discrepancy, following the conceptual study by King and Wesnousky (2007). Assuming that large earthquakes may penetrate below the interseismic seismogenic zone and that the corresponding amount is a function of earthquake size implies that fault width $W$ itself may not be a stationary fault property, but instead increases as a function of mainshock magnitude. From scaling relationships, based on observations of many earthquakes, it is clear that $W$ scales with magnitude (e.g., Wells and Coppersmith, 1994; Thingbaijam et al., 2017). Correspondingly, we hypothesize that the rate of increase of $W$ is changing (becoming distinctly smaller) once the background seismogenic depth is saturated. Therefore, the break in slope observed in magnitude-rupture area relationships (e.g., Hanks and Bakun, 2008) may well be an expression of a decreased rupture area growth rate (due to decreased rupture width growth rate; Fig. 5). As a consequence, the $W$ model may be a good approximation for earthquake scaling, while the apparent breakdown in scaling (because of increasing fault slip) may be the result of the depth penetration by large earthquakes below the seismogenic depth. Although the data presented here are insufficient to resolve these issues, we believe that our analysis may provide a pathway for future investigations in this direction. It highlights the potential importance of transient changes of the seismogenic zone for our understanding of earthquake source physics in that transient deepening of the seismogenic zone is positively correlated with mainshock magnitude, that is, mean slip in the region where transient deepening was observed.

\section{Data and Resources}

The Alaska earthquake catalog is provided by the Alaska earthquake center and accessed via National Earthquake Information Center's (NEIC's) Comprehensive Catalog (ComCat) web interface available at https://earthquake.usgs.gov/data/comcat/. The Japan earthquake catalog is provided by Japan Meteorological Agency, and the catalog is available at https://www.data.jma.go.jp/svd/eqev/data/bulletin/ index_e.html. Seismic catalogs from Turkey have been published by Bohnhoff et al. (2016) and may be found in the supplemental material to this article. Catalogs from California are hosted at https://scedc.caltech.edu/research-tools/alt-2011-dd-hauksson-yangshearer.html (southern California) and at http://www.ncedc.org/ ncedc/catalog-search.html (northern California). Kinematic source models were acquired from the SRCMOD database available at http://equake-rc.info/srcmod/. All web-based data sources (except Japan catalog, last accessed on April 2017) were last accessed on August 2019. The supplemental material contains an additional figure, in correspondence to Figure 4, for two different bin sizes (100event and 400-event bins, respectively) as well as the extracted seismic catalogs, used in this study.

\section{Acknowledgments}

This research project is supported with funding from King Abdullah University of Science and Technology (KAUST), Grant BAS/1/133901-01.

\section{References}

Allmann, B. P., and P. M. Shearer (2009). Global variations of stress drop for moderate to large earthquakes, J. Geophys. Res. 114, no. B01310, doi: 10.1029/2008JB005821.

Bohnhoff, M., M. Ickrath, and G. Dresen (2016). Seismicity distribution in conjunction with spatiotemporal variations of coseismic slip and postseismic creep along the combined 1999 Izmit-Düzce rupture, Tectonophysics 686, 132-145, doi: 10.1016/j.tecto.2016.07.029.

Bürgmann, R., and G. Dresen (2008). Crust and upper mantle: Evidence from rock mechanics, geodesy, and field observations, Annu. Rev. Earth Planet. Sci. 36, 531-567, doi: 10.1146/annurev.earth.36.031207.124326.

Byerlee, J. (1978). Friction of rocks, Pure Appl. Geophys. 116, 615-626. Cheng, Y., and Y. Ben-Zion (2019). Transient brittle-ductile transition depth induced by moderate-large earthquakes in southern and Baja California, Geophys. Res. Lett. 46, doi: 10.1029/ 2019 GL084315. 
Ellis, S., and B. Stöckhert (2004). Elevated stresses and creep rates beneath the brittle-ductile transition caused by seismic faulting in the upper crust, J. Geophys. Res. 109, no. B05407, doi: 10.1029/2003JB002744.

Hanks, T. C., and W. H. Bakun (2008). M-log A observations for recent large earthquakes, Bull. Seismol. Soc. Am. 98, no. 1, 490494, doi: 10.1785/0120070174.

Hauksson, E., W. Yang, and P. M. Shearer (2012). Waveform relocated earthquake catalog for southern California (1981 to 2011), Bull. Seismol. Soc. Am. 102, no. 5, 2239-2244, doi: 10.1785/0120120010.

King, G. C. P., and S. G. Wesnousky (2007). Scaling of fault parameters for continental strike-slip earthquakes, Bull. Seismol. Soc. Am. 97, no. 6, 1833-1840, doi: 10.1785/0120070048.

Lin, A., T. Maruyama, S. Aaron, K. Michibayashi, A. Camacho, and K. Kano (2005). Propagation of seismic slip from brittle to ductile crust: Evidence from pseudotachylyte of the Woodroffe thrust, central Australia, Tectonophysics 402, 21-35, doi: 10.1016/ j.tecto.2004.10.016.

Lin, G., P. M. Shearer, and E. Hauksson (2007). Applying a 3D velocity model, waveform cross-correlation, and cluster analysis to locate Southern California seismicity from 1981 to 2005, J. Geophys. Res. 112, no. B12309, doi: 10.1029/2007JB004986.

Mai, P. M., and G. C. Beroza (2000). Source-scaling properties from finite-fault rupture models, Bull. Seismol. Soc. Am. 90, 604-615.

Mai, P. M., and K. K. S. Thingbaijam (2014). SRCMOD: An online database of finite-fault rupture models, Seismol. Res. Lett. 85, no. 6, 13481357.

Magistrale, H. (2002). Relative contributions of crustal temperature and composition to controlling the depth of earthquakes in southern California, Geophys. Res. Lett. 29, no. 10, 1447, doi: 10.1029/2001GL014375.

Rolandone, F., R. Bürgmann, and R. M. Nadeau (2004). The evolution of the seismic-aseismic transition during the earthquake cycle: Constraints from the time-dependent depth distribution of aftershocks, Geophys. Res. Lett. 31, L23610, doi: 10.1029/2004GL021379.

Romanowicz, B. (1992). Strike-slip earthquakes on quasi-vertical transcurrent faults: Inferences for general scaling relations, Geophys. Res. Lett. 19, 481-484.

Schaff, D. P., G. H. R. Bokelmann, G. C. Beroza, F. Waldhauser, and W. L. Ellsworth (2002). High resolution image of Calaveras fault seismicity, J. Geophys. Res. 107, no. B9, 2186, doi: 10.1029/2001JB000633.

Scholz, C. H. (1982). Scaling laws for large earthquakes: Consequences for physical models, Bull. Seismol. Soc. Am. 72, no. 1, 1-14.

Scholz, C. H. (1988). The brittle-plastic transition and the depth of seismic faulting, Geol. Rundsch. 77, 319-328.
Scholz, C. H. (1994). A reappraisal of large earthquake scaling, Bull. Seismol. Soc. Am. 84, no. 1, 215-218.

Scholz, C. H. (2019). The Mechanics of Earthquakes and Faulting, Second Ed., Cambridge University Press, New York, New York, 471 pp.

Shaw, B. (2009). Constant stress drop from small to great earthquakes in magnitude-area scaling, Bull Seis. Soc. Am. 99, no. 2A, 871-875, doi: $10.1785 / 0120080006$.

Sibson, R. H. (1980). Transient discontinuities in ductile shear zones, J. Struct. Geol. 2, 165-171, doi: 10.1016/0191-8141(80)90047-4.

Sibson, R. H. (1986). Earthquakes and rock deformation in crustal fault zone, Annu. Rev. Earth Planet. Sci. 14, 149-175.

Stewart, C. A., and E. A. Miranda (2017). The rheological evolution of brittle-ductile transition rocks during the earthquake cycle: Evidence for a ductile precursor to pseudotachylyte in an extensional fault system, South Mountains, Arizona, J. Geophys. Res. 122, 10,643-10,655.

Thingbaijam, K. K. S., P. M. Mai, and K. Goda (2017). New empirical earthquake source scaling laws, Bull. Seismol. Soc. Am. 107, no. 5, 2225-2246, doi: 10.1785/0120170017.

Trepmann, C., and B. Stöckhert (2003). Quartz microstructures developed during non-steady state plastic flow at rapidly decaying stress and strain rate, J. Struct. Geol. 25, 2035-2051.

Turcotte, D. L., and G. Schubert (2002). Geodynamics, Second Ed., Cambridge University Press, New York, New York, 528 pp.

Wald, D. J., and T. H. Heaton (1994). Spatial and temporal distribution of slip for the 1992 Landers, California, earthquake, Bull. Seismol. Soc. Am. 84, no. 3, 668-691.

Waldhauser, F. (2009). Near-real-time double-difference event location using long-term seismic archives, with application to Northern California, Bull. Seismol. Soc. Am. 99, 2736-2848, doi: 10.1785/0120080294.

Waldhauser, F., and D. P. Schaff (2008). Large-scale relocation of two decades of Northern California seismicity using cross-correlation and double-difference methods, J. Geophys. Res. 113, no. B08311, doi: 10.1029/2007JB005479.

Wells, D. L., and K. J. Coppersmith (1994). New empirical relationships among magnitude, rupture length, rupture width, rupture area, and surface displacement, Bull. Seismol. Soc. Am. 84, no. 4, 974-1002.

Woessner, J., and S. Wiemer (2005). Assessing the quality of earthquake catalogues: Estimating the magnitude of completeness and its uncertainty, Bull. Seismol. Soc. Am. 95, no. 2, 684-698, doi: $10.1785 / 0120040007$.

Manuscript received 30 December 2019 Published online 10 June 2020 